\title{
ON THE EXISTENCE OF A DIFFERENTIAL COEFFICIENT
}

\author{
By W. H. Young, Sc.D., F.R.S., and Grace Chisholm Young, \\ PhIL.Doc. (Göttingen).
}

[Received July 21st, 1910._Read November 10th, 1910.]

1. The detailed account of the properties of derivates in Dini's Fondamenti per la teorica delle funzioni di variabili reali remained for some time the last word on the subject. The results there given that the upper and lower bounds of the derivates are the same in every interval, and that, in every interval, the derivates approach one another as near as we please,* might have seemed, in view of Weierstrass's. construction of a continuous function which possesses no differential coefficient anywhere of a final character. So the matter remained until Lebesgue proved that every function of bounded variation, and in particular every function with bounded derivates, possesses a finite differential coefficient everywhere, except at a set of content zero. The line of argument he adopted was in every respect a novel one. Besides the concept of the content of a set of points itself, which, though well known in the case of a closed set, required delicate constructive treatment in the case of an open set, Lebesgue made use of the still more abstract concepts of generalised integration and of Cantor induction. Neither of these latter concepts is used in the statement of the theorem, and it is not too much to say that, even at the present moment, few analysts, and still fewer mathematicians whose special interests are not analytical, are really at home with them. Some mathematicians, indeed, go so far as to regard a theorem as only plausible if the proof has involved the use of transfinite numbers.

It has long seemed to the authors that a proof of this fundamental and important result which used only those concepts which occur in its enunciation was a real desideratum, and recently attention was called at a meeting of this Society to the need in question. The present paper contains the complete solution of the problem here proposed.

* Dini, op. cit., German translation by Lüroth and Schepp, pp. 254-5.

† K. Weierstrass, Ablandlungen ans der Functionenlehre," pp. 97 seq. Sce also Hobson's Theory of Functions of a Real Tariable, p. 625. 
Other analogous results are known, but all of them involve in their very statement the concept of generalised integration. Thus they do not properly form part of the matter in hand. It may be noted, however, that, by means of the main result with regard to the existence of a differential coefficient, all the links in the chain of demonstration of these analogous results of Lebesgue's may be forged without the use of Cantor's numbers* and without, we may add, using any definition of generalised integration other than that based on monotone sequences. $t$

It will be noticed that we begin by proving a lemma analogous to the Heine-Borel theorem, but differing from it in two important respects: (a) the intervals obtained are non-overlapping, (b) certain of the points are left uncovered. This lemma, at least in the case when the set $S$ is the whole interval, might be deduced from Lebesgue's lemma concerning his chains of intervals, $\ddagger$ the proof of which involves Cantor's numbers. Moreover, conversely, Lebesgue's lemma is virtually contained in ours, though the application of it, without Cantor induction, would be difficult. We then use our lemma to prove the theorem concerning the existence of a differential coefficient in the case in which the derivates are bounded. Finally we deduce the more general from the special case by reasoning of an entirely different character, and involving still simpler considerations. $\S$ The paper terminates with two appendices, the first on the connection between Lebesgue's lemma and ours, and the second on the fundamental theorem of integration.

It may be added that the results of the paper, "On the Derivates of Non-differentiable Functions," $\|$ in the proof of which only the special case of Lebesgue's theorem was assumed, come, thanks to the present paper, to have a truth independent of the concepts of generalised integration and of Cantor's numbers; in other words, we can now assert that it has been demonstrated without the use of these concepts that there is necessarily a distinction of right and left in the values of the derivates of a nowhere differentiable function at a set of points which is everywhere dense and is of the first category, that the upper and lower

- There is one possible exception as to this statement, with which the authors are at present occupied. The reader is referred to a paper, by one of them, to be published in the October number of the Quarterly Journal of Mathenzatics, on "Functions of Bounded Variation," where a detailed account of the theorems in question is given.

† W. H. Young, "A New Method in the Theory of Integration," 1910, Proc. London Math. Soc.

+ "Functions of Bounded Variation," p. 73.

$\S$ This portion of the proof is repeated here for the convenience of readers from the paper quoted above.

". Messenger of Mathmatics, 1908, p. 65. 
bounds of the derivates at the points of this set are respectively $+\infty$ and $-\infty$, that at the remaining points of the interval both the upper derivates are $+\infty$ and both the lower derivates are $-\infty$, exception being made of at most another set of the first category, and that, finally, the function itself cannot in any interval have bounded variation.

2. Lemma.-If with each point of a closed set $S$ as left-hand end-point we have given an interval, or several intervals, we can find a finite number of these, nowhere overlapping, and such that the content of the sub-set of $S$ external to the chosen intervals is less than any pre-assigned positive quantity $e$.

Let $n$ be any chosen integer, and

$$
e_{1}>e_{2}>e_{3}>\ldots
$$

a monotone descending sequence of positive quantities, having zero as limit.

Let us elongate each of the given intervals $(x, x+h)$ by one $n$-th of its length, or less, in such a way that, instead of the single interval $(x, x+h)$, we have the set of intervals $(y, x+h)$, where

$$
x-\frac{h}{n} \leqslant y \leqslant x .
$$

Let $a$ be the left-hand end-point of the closed set $S$. Then the point $a$ determines the sub-set of the original intervals corresponding to elongated intervals having $a$ as left-hand end-point. Therefore, also, $a$ determines $e_{r}$, the first of the quantities $e_{i}$ of the above $e$-sequence which is not greater than all the original intervals of that sub-set.

Let us choose as the first of the intervals sought an original interval $d_{1}$ of the above mentioned sub-set, such that

$$
d_{1} \geqslant e_{r_{1}}
$$

the interval $d_{1}^{\prime}$, got by elongating* $d_{1}$ to the point $a$, is then one of the intervals we obtained at the commencement by elongation.

The points of $S$ which do not lie to the left of the right-hand endpoint of $d_{1}$ form a closed set, which we now proceed to treat in the same way as before. We thus obtain an integer $r_{2}$, and choose an interval $d_{2}$ of the original set, such that

$$
d_{2} \geqslant e_{r_{2}},
$$

and a corresponding interval $d_{2}^{\prime}$ of the elongated set, which will abut with

- $d_{1}^{\prime}$ may, of course, be identical with $d_{1}$. 
$d_{1}^{\prime}$ if the right-hand end-point of $d_{1}^{\prime}$ is a point of the closed set $S$, and otherwise will be such that between $d_{1}^{\prime}$ and $d_{2}^{\prime}$ there is no point of that closed set.

Continuing the process here indicated, we obtain a succession of the original intervals

$$
d_{1}, d_{2}, \ldots,
$$

and a corresponding succession of the elongated intervals

$$
d_{1}^{\prime}, d_{2}^{\prime}, \ldots,
$$

these latter leaving no point of $S$ between them uncovered.

We shall now shew that, after a finite number of such intervals have been chosen, the right-hand end-point of the last interval is within a distance $\frac{1}{2} e$ of the right-hand end-point $b$ of the closed set $S$.

For, if not, the countably infinite set of non-overlapping intervals $d_{i}$ determines a single limiting point $c$ to the right of all of them, but to the left of $b$. Since each $d_{i}$ contains a point of the set $S, c$ is a point of $S$, since that set is closed. Therefore there is an interval $\left(c, c+h_{c}\right)$ of the original set, and this determines the first integer $r_{c}$ such that

$$
e_{r_{c}} \leqslant h_{c} .
$$

Also, since $c$ is a limiting point of the intervals $d_{i}$, there is one of them, say $d_{k}$, inside the interval $\left(c-1 / n . h_{c}, c\right)$, so that the sub-set of the original intervals from which $d_{k}$ was chosen contains the interval $\left(c, c+h_{c}\right)$, which is $\geqslant e_{r_{c}}$. But $r_{i}$ is the smallest integer for which any interval of this sub-set $\geqslant e_{r_{k}}$; therefore $r_{k}$ is not greater than $r_{c}$. Hence

since $d_{k}$ was so chosen.

$$
e_{r_{c}} \leqslant e_{r_{k}} \leqslant d_{k},
$$

By precisely the same argument,

$$
e_{r_{c}} \leqslant d_{k+1} ;
$$

and so on. But this is manifestly absurd, since all the intervals $d_{k}, d_{k+1}, \ldots$ are internal to the interval $\left(c-1 / n . h_{c}, c\right)$. Thus the contingency here contemplated is impossible.

This proves the statement already made, that after a finite number, say $m$, of intervals have been chosen, the right-hand end-point of the last interval is within a distance $\frac{1}{2} e$ of $b$. Thus the elongated intervals $d_{1}^{\prime}, d_{2}^{\prime}, \ldots, d_{m}^{\prime}$ cover up the whole set $S$ to within a distance $\frac{1}{2} e$ of $b$. Also, by the principle of elongation, the fresh gaps left when we reduce the intervals $d_{i}^{\prime}$ again to $d_{i}$ (in doing which we may uncover some more of the points of $S)$, have a sum less than $\left(d_{1}+d_{2}+\ldots+d_{n n}\right) / n$, a fortiori 
less than $G / n$, where $G$ is the content of the whole set of original intervals. Thus our chosen intervals $d_{1}, d_{2}, \ldots, d_{m}$ cover up the whole of $S$ excepting a sub-set of content less than $\frac{1}{2} e+G / n$, which is less than $e$, provided $n$ was chosen greater than $2 G / e$. This, therefore, proves the lemma.

3. THEOREM.-If $f(x)$ is a monotone increasing function whose derivates are bounded, it has a differential coefficient, except at a set of content zero.

Let $G_{k}$ denote the set of points at which

$$
f^{+}(x)-f_{+}(x)>k \text {. }
$$

Then, if $k$ assumes in succession the values $A, \frac{1}{2} A, \frac{1}{4} A, \ldots$, where $A$ is the upper bound of the derivates of $f$, either there is some value of $k$ : for which $G_{k i}$ has a positive content or else every set $G_{k}$ has content zero, in which case the outer limiting set of the sets $G_{k}$ has content zero, that is, the set of all the points at which the right-hand derivates do not coincide has content zero.

Suppose then, if possible, that there is one of these values of $k$ such that $G_{k}$ has positive content. Now the set $G_{k}$ is itself the sum of the finite number of sets $H_{y, k}$ at which, besides (1), we have

$$
\frac{1}{2}(y-1) k \leqslant f_{+}(x)<\frac{1}{2} y k,
$$

$y$ denoting any positive integer up to that for which $\frac{1}{2}(y-1) k=A$. Hence at least one of these sets must have positive content. Suppose it is $H_{y, k}$, then there must be a closed sub-set of $H_{y, k}$ of positive content $S$.

Let $e$ be a sufficiently small positive quantity to satisfy the inequality

$$
\frac{1}{2} k S>e\{(y+1) k+2 A\},
$$

and let us divide the fundamental segment into equal compartments, so small that the sum of those which contain the points of the closed set $S$ is less* than $S+e$.

Now let $x$ be any point of the closed set $S$, and therefore an internal point, or a left-hand end-point of one of the compartments above mentioned. Then, by (1) and (2),

and

$$
\begin{gathered}
f_{+}<\frac{1}{2} y k, \\
\frac{1}{2}(y+1) k<f^{+}(x) .
\end{gathered}
$$

* This was the basis of Hankel's definition of the content of a closed set. Cp. Young's Theory of Sets of Points, p. 86. 
Therefore we can find two intervals $\left(x, x+h_{1}\right)$ and $\left(x, x+h_{2}\right)$ each inside the compartment determined above by the point $x$, and such that

and

$$
\begin{gathered}
f\left(x+h_{1}\right)-f(x)<\frac{1}{2} y k h_{1} \\
\frac{1}{2}(y+1) k h_{2}<f\left(x+h_{2}\right)-f(x) .
\end{gathered}
$$

By the lemma of $\$ 3$, we can choose out a finite number of nonoverlapping intervals in each case from the sets (6) and (7), so that the sum of the lengths of the chosen intervals is in each case greater than $S-e$, and the content of the points of $S$ not internal to them is less than $e$. In each case the set so chosen will leave over a finite set of intervals complementarily filling up the compartments containing the set $S$. Since the content of those compartments is less than $S+e$, it follows that the content of these complementary intervals is less than $2 e$, and therefore the increment of the function $f(x)$ over them is less than $2 e A$, since the derivates, and therefore the incrementary ratio, of $f$ are all $\leqslant A$.

Hence, if $P$ denote the increment of $f$ over the remaining compartments which contain no points of $S$ as internal points, and $P_{1}$ and $P_{3}$ respectively the increments over the chosen intervals from the sets (6) and (7), we have, since the sum of the increments from end to end of the fundamental segment is the same

$$
P+P_{2}<P+P_{1}+2 e A .
$$

But, by (6) and (7),

$$
\frac{1}{2}(y+1) k(S-c)<P_{2} ; \quad \text { and } \quad P_{1}<\frac{1}{2} y k(S+e),
$$

since the content of the chosen intervals is greater than $S-e$ and less than that of the compartments in which they lie, that is, less than $S+c$.

Hence

$$
\frac{1}{2}(y+1) k(S-e)<\frac{1}{2} y k(S+e)+2 e A,
$$

whence, a fortiori,

$$
\frac{1}{2} k S<e[(y+1) k+2 A] \text {, }
$$

which is in contradiction to (3).

Thus it is impossible that any one of the sets $G_{k}$ can have positive content, so that the set of points at which the upper and lower right-hand derivates are different has content zero.

Similarly, the set of points at which the upper and lower left-hand derivates are different has content zero; and again, similarly, the points at which the upper derivate on one side differs from the upper derivate on the other side has content zero. Hence, finally, the points at which there is not a differential coefficient form a set of zero content, which proves the theorem. 
CoR.-If $f(x)$ is any function having bounded derivates, it has a differential coefficient except at a set of content zero.

For, if the derivates are all greater than $-A$, the derivates of $f(x)+A x$ are all positive and bounded, so that by the theorem this function has a differential coefficient except at a set of content zero, whence the same is, of course, true for $f(x)$.

4. TheOREu.-Every monotone function possesses a finite differential coefficient, except at a set of content zero.

It is evidently sufficient to prove the theorem when all the derivates of the function are greater than some chosen positive quantity $A$. For, if the theorem is true for such a function, it is true for any monotone increasing function, since we change such a function into one whose derivates are greater than $A$ by adding $2 A x$, and a monotone decreasing function becomes monotone increasing by changing its sign.

Assuming, then, all the derivates to be greater than $A$, the function $y=F(x)$ is monotone increasing, without any tracts of invariability, and may be continuous or discontinuous. The inverse function $x=G(y)$ is therefore a continuous monotone increasing function of $y$, without or with tracts of invariability, and its derivates lie between $1 / A$ and zero, the former value excluded. Thus, by the theorem just proved, $G(y)$ possesses a differential coefficient except at a set of content zero of values of $y$.

Now the incrementary ratio of the function $G(y)$ has the same upper bound as the derivates, and is therefore less than $1 / A$. Describe round the exceptional set of values of $y$ a countable set of non-overlapping intervals of content $I$ as small as we please. Let $d$ be the length of one of these intervals. Then the values of $x$ corresponding to the values of $y$ at the ends of this interval necessarily differ by less than $d / A$, since the incrementary ratio is less than $1 / A$. Hence the values of $x$ corresponding to the exceptional values of $y$ are contained in a set of intervals whose sum is less than $I / A$, so that the content of the set of values of $x$ corresponding to the exceptional set of values of $y$ is less than $I / A$. Since $I$ may be as small as we please, this proves that the set of values of $x$ in question is also of zero content. Thus we have shewn that $G(y)$ possesses a differential coefficient except for values of $y$ corresponding to a set of values of $x$ of zero content. Hence $F(x)$ has a differential coefficient, finite or infinite, except for a set of values of $x$ of zero content.

Again, where the differential coefficient of $F(x)$ is infinite it is $+\infty$. Hence the points where this occurs form a set of zero content. For, if 
the content were $I$, we could surround the set in question by non-overlapping intervals, finite or countably infinite in number, such that the incrementary ratio of $F(x)$ over each such interval was greater than $B$, where $B$ is as large a positive quantity as we please, and therefore the sum of the corresponding increments of $F(x)$ would be greater than $B I$. Hence the net increment of the function over the whole interval, the function $F(x)$ being monotone increasing, would be greater than $B I$, which is only possible if $I$ is zero, since $B$ is as great as we please, and $F(x)$ is bounded.

Thus $F(x)$ possesses a difierential coefficient except at a set of content zero, and this differential coefficient is itself only infinite at a set of content zero, which proves the theorem.

Cor.-Every function of bounded variation possesses a finite differential coefficient except at a set of content zero.

For such a function is the difference of two monotone increasing functions.

\section{Appendix I.}

On the Virtual Identity of a Special Case of our Lemma with that of Lebesgue.

5. From our lemma we have the following corollary :-

CoR.-A countable non-overlapping set of the given intervals can be ruund such that ever!! point of the closed set $S$ is an internal or end-point of the selected intervals.

For, let the process described in the proof of the lemma be perforned in such a way that the extreme right-hand complementary interval is of length less than $G / n$, and let us proceed a stage further by repeating this process in each of the complementary intervals of the selected $d_{1}, d_{2}, \ldots, d_{m}$, and then again repeating the process, and so on. Then, either after a finite number of stages there is no point of $S$ left external to the intervals selected, in which case the statement is verified, or else we go on ad infinitum without ever arriving at such a final stage. With this latter hypothesis we can also shew, by a reductio ad absurdum, that the statement is true.

Suppose, in fact, if possible, that there is a point $c$ of the set $S$ which is at every stage external to the selected intervals. Then there is an interval $\left(c, c+h_{c}\right)$ of the original set, and this determines the first integer $r$ : such that

$$
e_{r_{c}} \leqslant h_{c} \text {. }
$$


At the end of the first stage the extreme right-hand gap, as well as each of the gaps introduced by reducing the elongated intervals $d_{1}^{\prime}, d_{2}^{\prime}, \ldots, d_{m}^{\prime}$ down again to $d_{1}, d_{2}, \ldots, d_{m}$, is less than $G / n$, where $G$ is the content of the original set of intervals. Therefore at the second stage the gaps introduced in the same way by cutting off the elongated parts will be less than $G / n^{2}$, and so on. Thus we can determine a stage from which onwards each of the gaps introduced in this way is less than $h_{\mathrm{c}} / n$.

Now, since $c$ is a point of $S$, but not internal to the selected intervals at any stage, it is at each stage internal to one of these gaps. Hence we see that, at the stage at which we have just arrived, the right-hand endpoint $P$ of one of the intervals selected lies inside the interval $\left(c-h_{c} / n, c\right)$. Hence, at the next stage, the nearest point of $S$ used as starting point will be either the point $c$ or lie between $c$ and $P$ inclusive, so that it is certainly a point of the interval $\left(c-h_{c} / n, c\right)$, whence it follows that the interval $\left(c, c+h_{c}\right)$ belongs to the subset from which the corresponding choice of, say, the interval $d_{k}$ is made. Hence

$$
e_{r_{c}} \leqslant d_{k},
$$

Similarly, at the next and each subsequent stage, there will be another interval selected of length $\geqslant e_{r_{c}}$, which is impossible if the total content $G$ of the given intervals is finite, as is assumed to be the case. Thus there can be no point of $S$ which is not internal to the selected intervals or an end-point of them.

When the set $S$ consists of all the points of a closed interval* $(a, b)$, the argument shews that no point $c$ of the closed set $S$ can be a righthand end-point of one of the selected intervals without being a left-hand end-point of another. Hence this special case is identical with that contemplated by Lebesgue, and the intervals form a Lebesgue chain reaching from $a$ to $b$.

On the other hand, the special case of our lemma follows immediately if we assume Lebesgue's lemma, viz.,

If with each point $x$ of the interval $(a, b)$ as left-hand end-point we have an interval or several intervals, we can find a chain of the intervals overlapping nowhere and such that every point of the interval $(a, b)$, except $b$, is an internal point or a left-hand end-point of an interval of the chain. $\dagger$

- It is obviously immaterial whether the point $b$ is included in the interval or not.

† This lemma is constantly used by Lebesgue, though never formally enunciated by him. Cp., for example, Leçons sur l'Intégration, p. 62. The statement given above is taken from "Functions of Bounded Variation." 
For in this case, by Cantor's theorem, the intervals are countable, being non-overlapping, and their lengths form a convergent series whose sum is $(a, b)$, since there is no point of $(a, b)$ external to them. Hence we can choose out a finite number of the intervals so that the sum of their lengths is greater than $(b-a-e)$. The sum of the complementary intervals is therefore less than $e$, which proves our lemma.

\section{APPENDIX II.}

On the Fundamental Theorem of Lebesgue Integration for a Function with Finite Derivates.

6. In this Appendix we propose to justify the statement, made in the introduction, as regards the fundamental theorem of integration in the case, first, of a function with bounded derivates, and, secondly, under someritat more general conditions.

ThEOREM.-A function with bounded derivates is the integral of any one of its derivates.

Consider the oscillating succession of functions

$$
\frac{f(x+h)-f(x)}{h} \text {. }
$$

Since this incrementary ratio for all values of $x$ and $h$ has the same upper and lower bounds as the derivates of $f$, the succession is bounded both above and below, and is therefore* semi-integrable both above and below, that is,

$$
\int_{a}^{x} f_{+}(x) d x \leqslant \operatorname{Llt}_{h=0} \int_{a}^{x} \frac{f(x+h)-f(x)}{h} d x \leqslant \int_{a}^{x} f^{+}(x) d x,
$$

taking $h$ to be positive.

But the succession of integrals converges to $f(x)-f(a)$, and the upper and lower right-hand derivates $f^{+}$and $f_{+}$are equal, except at a set of content zero, by the corollary in $\S 3$. Hence

$$
f(x)-f(a)=\int_{a}^{x} f_{+}(x) d x=\int_{a}^{x} f^{+}(x) d x,
$$

and, similarly, the equality with the integrals of the left-hand derivates, taking $h$ negative, follows.

Theorem.-A function whose derivates are bounded, except in the

- W. H. Young, "Term-by-term Integration of Oscillating Series," 1909 , Proc. London Math. Soc., Ser. 2, Vol. 8, p. 111. A slight and ubvious correction is needed in lines 14 and 15, and is given in the paper on "Semi-integrals and Oscillating Successions of Functions," by one of us, recently presented to the Society. The expression " semi-integrable" is introduced in this latter paper. 
neighbourhood of a countable set of points, is the integral of any one of its derivates provided it has bounded variation.

It follows, indeed, also from the theory of oscillating successions that the above succession is semi-integrable both above and below.* For $f(x)-f(a)$ is continuous, and the points in which the peak and chasm functions of the oscillating succession are infinite are countable.

In fact, since the upper bound of the derivates in ans interval is the same as that of the incrementary ratio, $t$ the points in the neighbourhood of which the derivates are unbounded above are the same as those in the neighbourhood of which the incrementary ratio $[f(x+h)-f(x)] / h,(0<h)$, regarded as a function of $(x, h)$, is unbounded above. These points evidently form a closed set, which is, by hypothesis, countable. $\ddagger$

But, $f(x)$ having bounded variation, its derivates agree except at a set of content zero and therefore, being summable, $\S$ have the same integral, which must therefore be equal to $f(x)-f(a)$. This proves the theorem.!

- Id., pp. 113, 114.

† So that the peak-function of the succession of incrementary ratios is the upper limiting function of the derivates.

$\ddagger$ [Added March 15th, 1911. - It follows from $\$ 3$ (Cor.), that a function whose derivates are bounded except at a closed set of content zero has a finite differential coefficient except at $a$ set of content zero. Hence, as at the end of $\$ 6$, we see that a function whose right (left) hand derivates are summable and are bon of any one of its derivates. For further results obtained without Cantor induction, see my "Note on the Fundamental Theorem of Integration" (1910), Proc. Camb. Phil. Soc., Vol. xvI, pp. 35-38.]

§ This follows as a corollary to the theorem of $§ 15$ of " Semi-Integrals," these Proceedings, p. 300 , since by that theorem the lower (upper) derivate of a monotone ascending (descending) function is summable.

|| This page has been modified during its passage through the press. 